\title{
A Reflectivity-Based GPR Signal Processing Methodology for Mapping Tree Root Systems of Street Trees
}

\author{
Livia Lantini ${ }^{*}$, Iraklis Giannakis ${ }^{*}$, Fabio Tosti*, Dale Mortimer ${ }^{\dagger}$, and Amir M. Alani* \\ *School of Computing and Engineering, University of West London (UWL), St Mary's Road, Ealing, London W5 5RF, UK \\ ${ }^{\dagger}$ Tree Service, London Borough of Ealing, Perceval House, 14-16 Uxbridge Road, Ealing, London W5 2HL, UK \\ Email: Livia.Lantini@uwl.ac.uk; Iraklis.Giannakis@uwl.ac.uk; Fabio.Tosti@uwl.ac.uk; MortimerD@ealing.gov.uk; \\ Amir.Alani@uwl.ac.uk
}

\begin{abstract}
Street trees are broadly acknowledged to be an essential asset of cities. Nonetheless, the growth of tree roots can lead to critical damage, such as the uplifting or cracking of road pavements and curbs, with severe implications on the infrastructure's safety. For this reason, the assessment and control of tree root systems' development have become crucial task in forestry and urban management. Within this framework, Ground Penetrating Radar (GPR) is widely recognised as an effective nondestructive testing (NDT) method for the monitoring and assessment of road infrastructures. This study aims to demonstrate the capability of GPR in mapping the root system's architecture of street trees. To this end, a GPR system equipped with a $700 \mathrm{MHz}$ central frequency antenna was employed to investigate the area around a street tree, located in the vicinity of a flexible pavement structure. A novel processing framework based on the analysis of the signal reflectivity was implemented, in order to automatically identify the reflections from the pavement layers and apply dedicated advanced signal processing techniques. A multistage data processing methodology was employed to map the tree root system architecture. Finally, information on the mass density of roots at different depths was also provided. Results have proven the potential of the proposed methodology to achieve an automatic detection and mapping of roots under road pavements.
\end{abstract}

Keywords - Ground Penetrating Radar (GPR), pavement damage, road safety, street trees, tree root mapping, tree root mass density

\section{INTRODUCTION}

Street trees are an integral part of the urban environment. Beyond the merely ornamental role they covered in the past, trees nowadays play an active role for the welfare and economy of the city, as well as for the social life of its inhabitants [1,2]. Nonetheless, the coexistence of trees and built environment is

Acknowledgements: The authors would like to express their sincere thanks and gratitude to the following trusts, charities, organisations and individuals for their generosity in supporting this project: Lord Faringdon Charitable Trust, The Schroder Foundation, Cazenove Charitable Trust, Ernest Cook Trust, Sir Henry Keswick, Ian Bond, P. F. Charitable Trust, Prospect Investment Management Limited, The Adrian Swire Charitable Trust, The John Swire 1989 Charitable Trust, The Sackler Trust, The Tanlaw Foundation, and The Wyfold Charitable Trust. This paper is dedicated to the memory of Jonathan West, a friend, a colleague, a forester, a conservationist and an environmentalist who died following an accident in the woodland that he loved. often difficult. In particular, with regard to road infrastructures, one of the main causes of pavement degradation in urban areas is due to the uncontrolled growth of street tree roots. These can cause severe damage and deformation to pavements and curbs, such as uplifting and cracking [3-5], which can significantly affect road safety conditions [6]. It is also important to emphasise that poor design choices and lacks of proper road maintenance activities can worsen the overall status of the infrastructure [7].

The selection of species not suitable for growing in confined environments, due to the trunk size at maturity $[8,9]$ or the root system spread compared to the available volume of soil $[10,4]$, can cause damage to the tree itself and to the road surface. However, the soil volumes required for normal tree growth are generally not available in an urban environment [2]. It is also important to emphasise that, while tree root systems need porous soils to prosper and expand freely [11], road engineering requirements for the construction of an infrastructure impose a load-bearing base, to support high traffic loads [12]. These compaction levels prevent the roots from penetrating the soil, and also limit their access to air, water and nutrients, inhibiting their growth and development $[13,14]$. However, tree roots can find a more favourable condition for growth at the interface between the pavement and the soil, or between the various layers of the pavement itself [15-18]. This is due to the pavement's difference in temperature between day and night, which allows condensation to form below the pavement structure [17]. This optimal environment attracts roots, which quickly absorb moisture [16], resulting in a humidity gradient that further encourages root growth and thickening [17]. This process can result in severe damages of the pavement structure and surface [18].

For this reason, locating and tracing tree roots below a road infrastructure is vital, in order to monitor the state of the pavements and plan adequate maintenance. To this end, Ground Penetrating Radar (GPR) is widely recognised to be a reliable non-destructive testing (NDT) method for locating buried objects and imaging the subsurface. Furthermore, it is a proven and efficient method for investigating road infrastructures [19], ranging from the evaluation of pavement layer thicknesses [20, 21], to damage assessment in hot-mix asphalt (HMA) layers 
[22], load-bearing layers and subgrade soils [23]. Finally, recent advances in the use of GPR include the assessment and monitoring of tree root systems [24-26].

\section{AIM AND OBJECTIVE}

This research aims to demonstrate the capability of midrange frequency GPR antenna systems in providing threedimensional mapping of tree root systems in urban areas (beneath the road surface).

To achieve this aim, the main objective of this research is to improve upon existing methods for automatically locating and mapping tree roots in high-reflectivity areas, such as road infrastructures. A further objective is the provision of root density maps at different depths, to facilitate the interpretation of local variations of the root concentration, especially at the transition areas between the natural soil and the road pavement structure.

\section{MEthodology}

\section{A. Test Site and Equipment}

The survey was carried out in Gunnersbury Park, Ealing, London (United Kingdom). A number of 21 semi-circular scans were performed around the investigated tree, starting $0.50 \mathrm{~m}$ from the bark and spaced $0.30 \mathrm{~m}$ from one another. Therefore, an overall area of $175.67 \mathrm{~m}^{2}$ was surveyed around the tree, with an outer radius of $7.66 \mathrm{~m}$ and an inner radius of $1.66 \mathrm{~m}$. This particular setup was designed to include a road section covered with a flexible pavement structure within the investigated area, to gather data both from natural soil and asphalt-covered road surface (Fig. 1).

The survey was carried out using the Opera Duo groundcoupled GPR system, manufactured by IDS GeoRadar (Part of Hexagon). The system is equipped with $700 \mathrm{MHz}$ and $250 \mathrm{MHz}$ central frequency antennas. Data were collected using a time window of $80 \mathrm{~ns}$, discretised across 512 samples. The horizontal resolution was set to $3.06 \times 10^{-2} \mathrm{~m}$. In order to achieve the optimal effective resolution, only data collected using the 700 $\mathrm{MHz}$ antenna were analysed. This choice was due to the need to achieve a depth of investigation such as to include the whole tree root system, without overly affecting the signal resolution.

\section{B. Data Processing Methodology}

\section{Signal Processing Stage}

The need to automatically detect horizontal reflections linked with the horizontal multi-layered configuration of the road pavement has led to the development of a multi-stage signal processing algorithm. To this purpose, a first stage of data cleaning was carried out, to increase the likelihood of root detection and reduce false alarms by removing unwanted reflections and increasing the signal-to-noise ratio (SNR). Subsequently, an innovative method based on the analysis of data reflectivity was employed, in order to automatically identify the geometry of the pavement layers. The rationale behind the use of this latter step is due to the horizontal reflections affecting only part of the dataset (i.e. the section with the road pavement layers). This prevents the use of the same signal processing

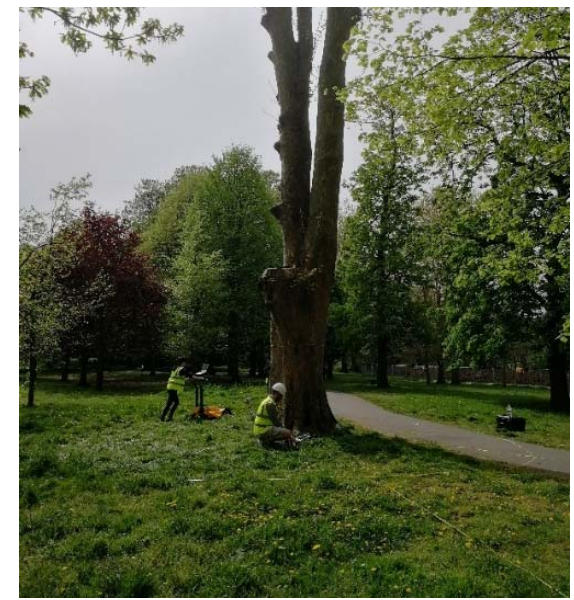

Fig. 1. The investigated area

methodology over the whole dataset and allows to use dedicated signal processing frameworks as per the structure investigated. Based on the result of the reflectivity analysis, it was in fact possible to limit the application of the Singular Value Decomposition (SVD) filter to concerning areas. This filter is particularly effective in reducing the effects of the horizontal features (e.g. the layers of a road pavement) from GPR data. The concept on which an SVD filter is based is that a GPR image can be decomposed into different sub-images (eigenimages), each of which contains part of the primary image information with increasing correlation. Signal components such as ringing noise or reflections given by road pavements are accounted as highly correlated attributes, as opposed to the response of the target, which is a less correlated component. The division into subimages, therefore, allows for a targeted elimination of the horizontal features, without affecting the target response and increasing the SNR.

The steps of the signal processing stage are as follows:

- Preliminary data processing: a sequential application of i) zero-offset removal, ii) bandpass filtering and iii) time-varying gain was carried out;

- Analysis of the signal reflectivity: each B-scan was tested to evaluate changes in the signal reflectivity, so as to highlight the presence of the road pavement's different layers;

- Targeted application of SVD filter: in the areas identified in the previous step, the SVD filter was applied to eliminate horizontal reflections without altering the reflections due to the target of the investigation (i.e. tree roots).

\section{Tree Root Tracking Algorithm}

Following the signal processing stage, a semi-automatic algorithm was employed in order to locate and track the tree roots based on the revised B-scans. Data are analysed and processed by means of an iterative procedure, in order to to compare the amplitude values at different positions of the threedimensional domain and find a spatial correlation between them. The phases of the iterative procedure are as follows: 
- Target detection: the amplitude values are compared with a preset threshold value throughout the analysed domain, to identify the candidate reflections from the tree roots;

- Spatial correlation: a potential correlation is sought between the identified targets, based on their position in the three-dimensional space;

- Tracking of the roots: vectors are used to connect the targets, thus creating the spatial development of the individual roots;

- $\quad$ Reconstruction of the root system architecture in the 3$D$ domain: the vectors are placed in a three-dimensional environment, therefore creating an inclusive rendering of the root system.

\section{Tree Root Density Estimation}

In the last phase, root density is estimated based on the reconstructed root system architecture. Employing a polynomial fitting, the root path was better approximated in a continuous domain and, as a result, it was possible to calculate the length of each root. This led to the definition of an index for the calculation of the root density, which evaluates the length of the roots in a reference volume (in this case equal to $0.3 \mathrm{~m} \times 0.3 \mathrm{~m}$ $\times 0.3 \mathrm{~m})$

$$
d=\frac{\sum_{i=1}^{n} L_{i}}{V}
$$

where $d$ is the density $\left[\mathrm{m} / \mathrm{m}^{3}\right], n$ is the number of roots contained in a reference unit of volume $\left[\mathrm{m}^{3}\right]$ and $L_{i}$ is the length of the root $[\mathrm{m}]$.

\section{MAIN RESUlts AND SHORT Discussion}

The use of a pre-processing phase on the GPR data allowed for an effective reduction of the noise and a significant improvement in target detection. As an example, Fig. 2 shows a longitudinal B-scan collected along the seventh scanline. Fig. 2(a) shows the B-scan before the application of the processing, whereas Fig. 2(b) shows the B-scan after the application of the main data processing techniques. The presence of road layers is clearly visible on the left side of the B-scans. In this regard, the analysis of the signal reflectivity has shown effectiveness in automatically detecting the presence of a road structure (Fig. 2(c)), thus allowing for a targeted application of the SVD filter (Fig. 2(d)).

Subsequently, the algorithm for the analysis of the spatial correlation and the automatic tracking of the roots in threedimensional space was applied to the processed data. Fig. 3 shows the result of this procedure in a 2-D planar view (a) and in a 3-D environment (b). To simplify the interpretation of the results, the shallow-buried roots (i.e. within the first $25 \mathrm{~cm}$ of soil) have been represented in green, while the deeper roots (i.e., beyond a depth of $25 \mathrm{~cm}$ from the soil surface) have been highlighted in brown. Results clearly show the potential of the algorithm to reconstruct a comprehensive rendering of the root system. For the sake of consistency, the point coordinates were double-checked against the positions of the focused targets, through the analysis of the single B-scans.

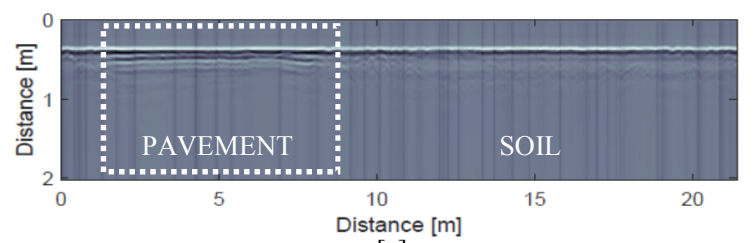

[a]

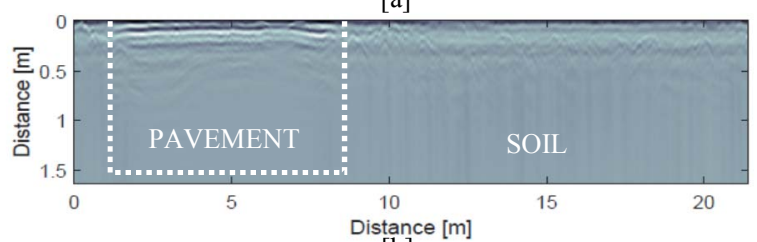

$[\mathrm{b}]$
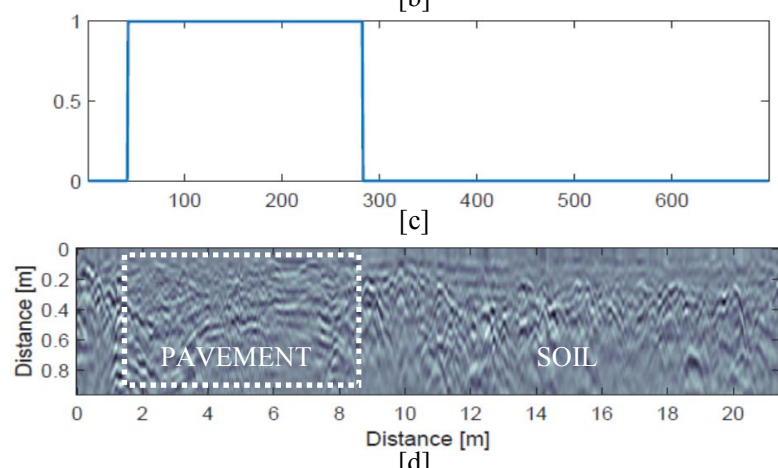

[d]

Fig. 2. An example of GPR data pre-processing. a) Unprocessed B-scan, b) B-scan after main processing techniques, c) analysis of the signal reflectivity, showing a maximum value at the road pavement section, and d) B-scan after a targeted application of SVD filter

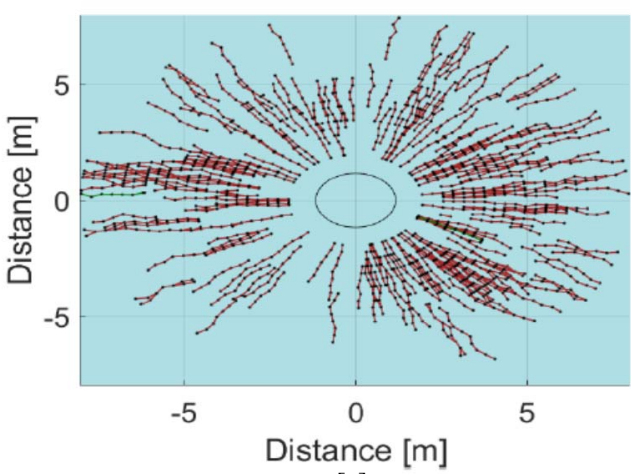

[a]

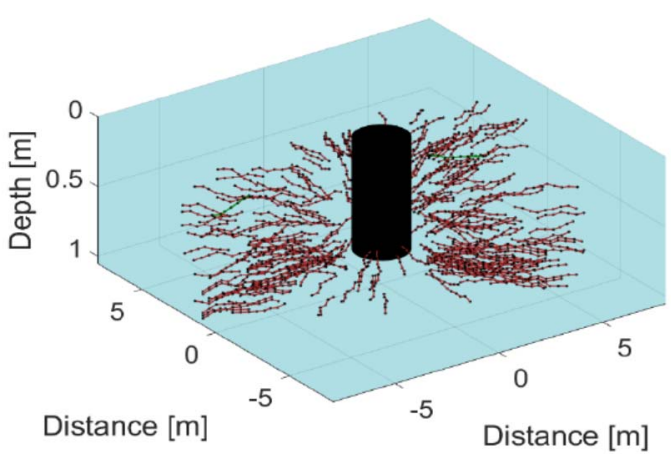

[b]

Fig. 3. Reconstruction of the root system architecture of the surveyed tree: a) $2 \mathrm{D}$ planar view and b) $3 \mathrm{D}$ reconstruction 


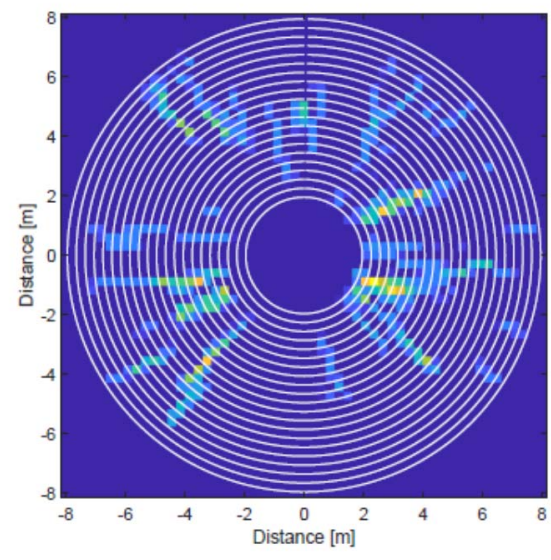

Fig. 4. Root density map, related to a depth from $0.50 \mathrm{~m}$ to $0.60 \mathrm{~m}$

At the final stage, the root density at different depths was examined using Eq. (1). The investigated domain was divided into reference volumes and analysed to estimate the total length of roots per reference unit. Fig. 4 presents an example of the outcomes of this processing stage, in the form of a density map at a depth range from $0.50 \mathrm{~m}$ to $0.60 \mathrm{~m}$.

\section{CONCLUSION}

This study reports a demonstration of the potential of Ground Penetrating Radar (GPR) to detect tree roots growing underneath road infrastructures using an automatic data processing methodology. The primary aim of the study was to demonstrate the GPR potential in providing effective three-dimensional mapping of the root systems of street trees located under the road surface. A multi-stage interpretation algorithm was presented, aiming at reconstructing the patterns of tree roots using GPR. The proposed methodology is based on the collection of circular measurements expanding radially from the tree trunk. Initially, a pre-processing step was applied to eliminate noise-related features and enhance the response from the real targets. Subsequently, the analysis of the signal reflectivity was carried out to automatically identify the presence of the pavement layers and apply a Singular Value Decomposition (SVD) filter in a targeted way. Afterwards, a tracking algorithm was used in order to locate and automatically outline viable root paths. Lastly, the identified roots were expressed through the use of continuous functions, and the root density was analytically derived. A case study is presented, in which the proposed methodology was successfully applied to a tree, with the root system partially located underneath a road infrastructure. Both shallow (i.e. within the first $25 \mathrm{~cm}$ of soil) and deep (i.e. below $25 \mathrm{~cm}$ from the surface of the soil) root structures were successfully identified, supporting the statement that GPR is an effective NDT method for the assessment of street trees' roots.

\section{REFERENCES}

[1] G. S. Seamans, "Mainstreaming the environmental benefits of street trees," Urban Forestry \& Urban Greening, vol. 12, no. 1, pp. 2-11, 2013.
[2] J. Mullaney, T. Lucke and S. J. Trueman, "A review of benefits and challenges in growing street trees in paved urban environments," Land. and Urban Plan., vol. 134, pp. 157-166, 2015.

[3] R. W. Day, "Damage of structures due to tree roots," Jour. of perf. of const. fac., vol. 5, no. 3, pp. 200-207, 1991.

[4] J. K. Francis, B. R. Parresol and J. M. de Patino, "Probability of damage to sidewalks and curbs by street trees in the tropics," Jour. of Arb., vol. 22, 1996.

[5] S. M. Blunt, "Trees and pavements - are they compatible?," Arb. Jour., vol. 31, no. 2, pp. 73-80, 2008.

[6] F. Tosti, L. Bianchini Ciampoli, M. G. Brancadoro and A. Alani, "GPR applications in mapping the subsurface root system of street trees with road safety-critical implications," Adv. in Transp. Stud., vol. 44, 2018.

[7] K. D. Coder, "Tree roots and infrastructure damage," Univ. of Geor. Coop. Ext. Ser. For. Res., 1998.

[8] J. A. Wagar and P. A. Barker, "Tree root damage to sidewalks and curbs," Jour. of Arb., vol. 9, no. 7, pp. 177-181, 1983.

[9] L. R. Costello and K. S. Jones, Reducing infrastructure damage by tree roots: a compendium of strategies, West. Chap. of the Int. Soc. of Arb., 2003.

[10] P. Lindsey and N. Bassuk, "Redesigning the urban forest from the ground below: A new approach to specifying adequate soil volumes for street trees," Arb. Jour., vol. 16, no. 1, pp. 25-39, 1992.

[11] J. Grabosky, E. Haffner and N. Bassuk, "Plant available moisture in stone-soil media for use under pavement while allowing urban tree root growth," Jour. of Arb., vol. 35, no. 5, p. 271, 2009.

[12] J. Grabosky, N. Bassuk, L. Irwin and H. Van Es, "Pilot Field Study of structural soil materials in pavement profiles," in The Land. Bel. Gr. II: Proc. of an Int. Work. on Tree Root Dev. in Urb. Soils, San Francisco, CA, 1998.

[13] F. C. Loh, J. C. Grabosky and N. L. Bassuk, "Growth response of Ficus benjamina to limited soil volume and soil dilution in a skeletal soil container study,” Urb. For. \& Urb. Gr., vol. 2, no. 1, pp. 53-62, 2003.

[14] S. R. Tracy, C. R. Black, J. A. Roberts and S. J. Mooney, "Soil compaction: a review of past and present techniques for investigating effects on root growth," Jour. of the Sci. of Food and Agr., vol. 91, no. 9, pp. 1528-1537, 2011.

[15] J. Kopinga, "Aspects of the damage to asphalt road pavings caused by roots," in The Land. Bel. Gr.. Proc. of an Int. Work. on Tree Root Dev. in Urb. Soils, Champaign, IL, 1994.

[16] J. A. Wagar and A. L. Franklin, "Sidewalk effects on soil moisture and temperature," Jour. of Arb., vol. 20, 1994.

[17] T. B. Randrup, E. G. McPherson and L. R. Costello, "A review of tree root conflicts with sidewalks, curbs, and roads," Urb. Ecos., vol. 5, no. 3, 2001.

[18] B. C. Nicoll and A. Armstrong, "Development of Prunus root systems in a city street: Pavement damage and root architecture," Arb. Jour., vol. 22, no. 3, pp. 259-270, 1998

[19] T. Saarenketo and T. Scullion, "Road evaluation with ground penetrating radar,” Jour. of App. Geo., vol. 43, no. 2-4, pp. 119-138, 2000.

[20] I. L. Al-Qadi and S. Lahouar, "Measuring layer thicknesses with GPR-Theory to practice," Constr. and Build. Mat., vol. 19, no. 10, pp. 763-772, 2005.

[21] A. Loizos and C. Plati, "Accuracy of pavement thicknesses estimation using different ground penetrating radar analysis approaches," NDT\&E Int., vol. 40, no. 2, pp. 147-157, 2007.

[22] I. L. Al-Qadi, S. Lahouar and A. Loulizi, "In situ measurements of hot-mix asphalt dielectric properties,” NDT\&E Int., vol. 34, no. 6, pp. 427-437, 2001.

[23] A. Benedetto, F. Tosti, B. Ortuani, M. Giudici and M. Mele, "Mapping the spatial variation of soil moisture at the large scale using GPR for pavement applications," Near Surf. Geoph., vol. 13, no. 3, pp. 269-278, 2015.

[24] A. M. Alani, L. Bianchini Ciampoli, L. Lantini, F. Tosti and A. Benedetto, "Mapping the root system of matured trees using ground penetrating radar," in 2018 17th Int. Conf. on Gr. Pen. Rad. (GPR), Rapperswil, Switzerland, 2018.

[25] L. Lantini, R. Holleworth, D. Egyir, I. Giannakis, F. Tosti and A. Alani, "Use of ground penetrating radar for assessing interconnections between root systems of different matured tree species," in 2018 IEEE Int. Conf. on Metr. for Arch. and Cult. Her., Cassino, Italy, 2018.

[26] A. M. Alani and L. Lantini, "Recent advances in tree root mapping and assessment using non-destructive testing methods: a focus on ground penetrating radar," Surv. in Geoph., pp. 1-42, 2019 